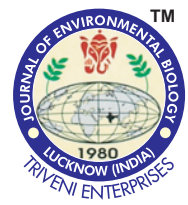

\title{
Altered immunohistochemical distribution of hippocampal interneurons following traumatic brain injury
}

\begin{abstract}
Authors Info
Y.H. Yu, J.H. Kang, K.H. Lee, D.Y. Yoo, D.K. Park and D.S. Kim*

Department of Anatomy, Soonchunhyang University, Cheonan, 31151, Republic of Korea

*Corresponding Author Email : dskim@sch.ac.kr

\section{Edited by}

Professor Seongho Ryu

Reviewed by

Professor Ki-Yeon Yoo

Professor Dae-Won Kim

\section{Abstract}

Aim: The aim of this study was to determine whether alteration of interneurons in the hippocampus might play a role in neuronal damage caused by brain trauma and whether immunohisto chemical expressions in the hippocampus might change during the adaptive stage as early time point following traumatic brain injury (TBI).

Methodology: For the study, we used Sprague-Dawley (SD) rats and manufactured TBI animal model by creating cryogenic injury to specific brain tissue. Thereafter, immunohisto chemical approaches were utilized to determine parvalbumin (PV) and calbindin D-28K (CB) expression in the hippocampus following brain trauma in a time course. All hippocampal tissues were analyzed qualitatively and quantitatively using immunoreactivity, cell counting and densitometry. Statistical significance was determined by one-way ANOVAand Bonferroni's test.

Results: At early time period following $\mathrm{TBI}$, both $\mathrm{PVand} C \mathrm{CB}$ immunoreactivities decreased in the lesioned hippocampus. However, their expression levels were recovered to control levels as time passed by. On the other hand, PV immunoreactivity in contralateral hippocampus was transiently reduced whereas CB expression remained unchanged.

Interpretation: Results of this study revealed that altered distribution of interneuronal populations in the hippocampus might contribute to neuronal loss induced by abnormality of inhibitory neurotransmission at early time period following brain damage, thus leading to the development of epileptogenesis in patients with TBI.

Key words: Calbindin D-28K, Epileptogenesis, Parvalbumin, Post-traumaticepilepsy, Traumatic brain injury

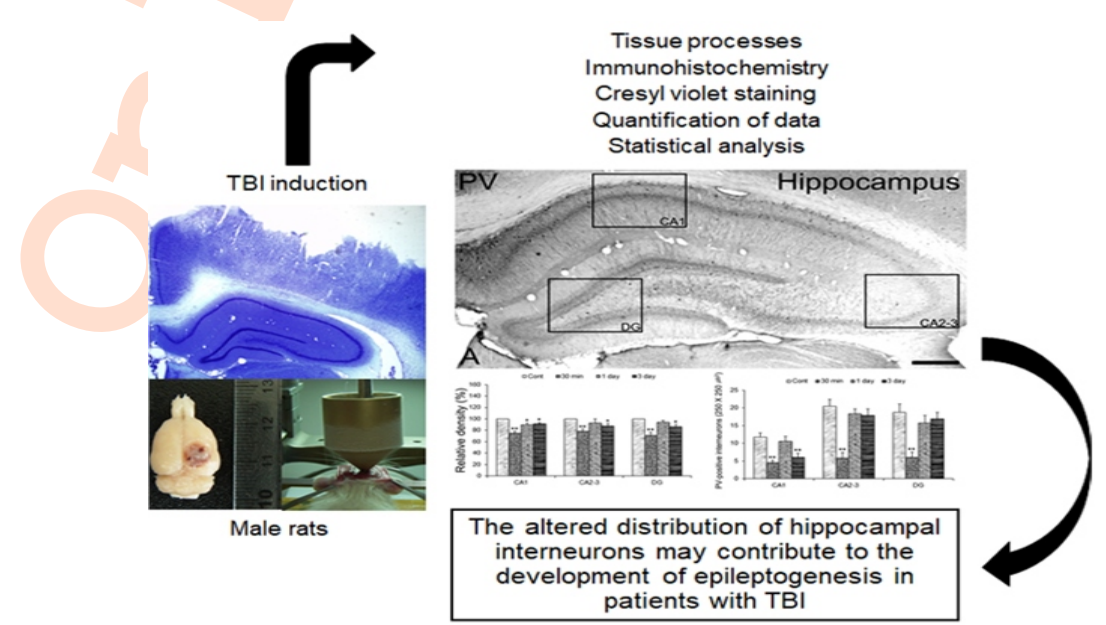

How to cite : Yu, Y.H., J.H. Kang, K.H. Lee, D.Y. Yoo, D.K. Park and D.S. Kim: Altered immunohistochemical distribution of hippocampal interneurons following traumatic brain injury. J. Environ. Biol., 40, 833-840 (2019). DOI : http://doi.org/10.22438/jeb/40/5(SI)/SI-02
\end{abstract}




\section{Introduction}

It is well known that traumatic brain injury (TBI) is accompanied by a delayed pathophysiologic change in degenerated neurons when the brain tissue is directly damaged by external factors (Gaetz, 2004). These brain injuries can degrade various cognitive functions and impair motor ability of patients. In some patients, TBI may cause recurrent epileptic seizures due to sequela of brain damage (Kharlamove et al., 2011). Although consequences of TBI differ by individuals, more than $50 \%$ of patients with recurrent epileptic seizure can progress to post-traumatic epilepsy (PTE) within oneyear (Willmore and Ueda, 2009). In addition, the morbidity rate of epileptic patients increases with the severity of brain damage (Weiss et al., 1983, 1986).

On the other hand, some previous studies have demonstrated that the cause of epilepsy is due to excessive enhancement of neuronal excitability caused by abnormal inhibitory neurotransmissions (Guerrini, 2001; Meldrum, 2002; Rozycka and Trzeciak, 2003). Thus, the main cause of pathophysiological characteristics of epilepsy might be attributed to abnormal production of inhibitory neurotransmitter such as $y$ aminobutyric acid (GABA). In addition, significantly downregulated GABAergic interneuron due to diverse brain damage effects can result in declined control of neuronal excitability, thus leading to epileptogenesis following brain trauma (De Lanerolle et al., 1989). However, in, several studies a variety of experimental animal models have shown that GABAergic interneuron is increased in epileptic animals as compared to normal group. Moreover, "disinhibition theory" states that epilepsy occurs due to inhibition of GABA secretion by formation of many inhibitory synapses in GABAergic interneuron (Peterson, 1987). Despite these efforts, the basic mechanism of epileptogenesis following brain trauma has not been clarified yet, although epilepsy caused by TBI accounts for more than $5 \%$ of all epilepsy patients and more than $20 \%$ of symptomatic epilepsy patients (Pagni and Zenga, 2005; Garga and Lowenstein, 2006; Mani and Barry, 2006).

Calcium binding proteins (CBPs), including parvalbumin (PV) and calbindin D-28k (CB), that regulate the intracellular calcium ion in response to neuronal hyperexcitability are well known as specific markers for denaturation of various neurons (Fritschy et al., 1999; Sutch et al., 1999). These results seem to reveal that the functionalities of various GABAergic interneurons play an important role in the pathogenesis of epilepsy following $\mathrm{TBI}$, suggesting that CBPs might be altered in this process. Therefore, the present study was carried out to determine the effects of GABAergic interneurons on the development and spread of epilepsy after TBI, using an experimental animal model at early time stage. Basic medical data was also examined to determine the role and function of these factors in the pathogenesis of epilepsy, following TBI.

\section{Materials and Methods}

Experimental animals: All experiments utilized the progeny of Sprague-Dawley rats obtained from the Experimental Animal Center, Soonchunhyang University (Cheonan, South Korea). All animals were provided access to a commercial diet and water ad libitum under controlled temperature, humidity and lighting conditions (light/dark cycle $12: 12$, and $22 \pm 2{ }^{\circ} \mathrm{C}, 55 \pm 5 \%$ ). All animal protocols were approved by the Administrative Panel on Laboratory Animal Care of Soonchunhyang University (permit No. SCH15-0002). All efforts were made to avoid suffering of rats and minimize the number used during the experiments.

Induction of traumatic brain injury: The cryogenic TBI animal model was used as described in previous study (Siren et al., 2006) with some modification. Briefly, ten-week-old male Sprague-Dawley rats were anaesthetized under an intraperitoneal injection of ketamine $\left(90 \mathrm{mg} \mathrm{kg}^{-1}\right)$ and xylazine (4 $\left.\mathrm{mg} \mathrm{kg}^{-1}\right)$. The frotoparietal skull was exposed through a scalp incision under stereotaxic frame and a cryogenic lesion was placed on the right frontoparietal cortex (unilateral lesions) or on both frontoparietal cortices (bilateral lesions). For cryogenic lesions, a cone-shaped copper cylinder with tip diameter of $2 \mathrm{~mm}$ was cooled with liquid nitrogen $\left(-183^{\circ} \mathrm{C}\right)$. The tip was stereotaxically placed in direct contact with the exposed frontoparietal skull and kept in place for $60 \mathrm{sec}$. Sham-operated animals underwent the same procedure without cooling the metal cone.

Tissue processes: Anesthetized animals (urethane $1.5 \mathrm{mg} \mathrm{kg}^{-1}$ ) were perfused transcardially with PBS, followed by fixing with $4 \%$ paraformaldehyde in $0.1 \mathrm{M} \mathrm{PB}$ for different time periods for a time course study (30 min, 1, 3, 6, 12, $24 \mathrm{hr}$ and 3, 5, 7 days after cryogenic lesion and control). Brains were removed and postfixed in the same fixative for $4 \mathrm{hr}$ and rinsed in PB containing $30 \%$ sucrose at $4{ }^{\circ} \mathrm{C}$ for 2 days. Thereafter, tissues were frozen and sectioned with a cryostat to $30 \mu \mathrm{m}$ thickness. Consecutive sections were collected in six-well plates containing PBS. For cell counts, every sixth section in the series throughout the entire hippocampus of the same animal were used. To determine neuronal survival in hippocampus, counter staining of Nissle bodies was performed with cresyl violet solution after cryogenic lesion.

Immunohistochemistry: Immunohistochemical procedures were performed according to published studies (Kim et al., 2008) with some modifications. Briefly, sections were first incubated with $3 \%$ bovine serum albumin in PBS for $30 \mathrm{~min}$ at room temperature. Sections were then incubated with the rabbit antiparvalbumin (PV) IgG and/or the rabbit anti-calbindin D-28k (CB) $\lg \mathrm{G}(1: 1,000$, Chemicon, Temecula, CA, USA) primary antibodies in PBS containing $0.3 \%$ triton $\mathrm{X}-100$ overnight at room temperature. The sections were washed three times with PBS for 10 min each and sequentially incubated with biotinylated goat 
anti-rabbit lgG (Vector, Burlingame, CA, USA) and ABC complex (Vector, USA) diluted 1:200 in the same solution as primary antiserum. Between incubations, tissues were washed with PBS three times for $10 \mathrm{~min}$ each. These sections were then visualized with 3,3'-diaminobenzidine in $0.1 \mathrm{M}$ Tris buffer and mounted on gelatin-coated slides. Immunoreactions were observed under a Leica DMRB microscope (Germany), and images were captured using an Olympus DP72 digital camera and a DP2-BSW microscope digital camera software. To establish the specificity of immunostaining, a negative control test was carried out with preimmune serum instead of primary antibody. The negative control resulted in the absence of immunoreactivity in any structures.

Quantification of data and statistical analysis: For quantification of each immunostaining, cell count was determined in the hippocampus according to previous studies (Kim et al., 2007a; An and Kim, 2011). Briefly, immunostaining images (10 sections per animals) were captured in the same region $(500 \times 500$ $\mu \mathrm{m})$. Images were sampled from at least five different points within each hippocampal section. Thereafter, the number of each primary antibodies-positive cells within the sampled image was counted. All immunoreactive cells were counted regardless the intensity of labeling. Cell counts were performed by two different investigators who were blinded to the classification of tissues. In addition, hippocampal subregions (CA1, CA2-3 and DG) were delineated with a $2.5 \times$ objective lens. Each image was normalized by adjusting the black and white range of the image using Adobe PhotoShop v. 8.0. Thereafter, 10 areas per rat (250 $\mu \mathrm{m}^{2}$ for each area) were selected. Intensity measurements are presented as mean number of a 256 gray scale using NIH Image 1.59 software. Values of background staining were obtained from the corpus callosum. Optical density values were corrected by subtracting the average values of background noise obtained from five image inputs. All data obtained from the quantitative measurements were analyzed using one-way ANOVA to determine statistical significance. Bonferroni's test was used for post hoc comparisons. $P$-value less than 0.05 and 0.01 was considered statistically significant (Kim etal., 2007b).

\section{Results and Discussion}

Previous studies have reported that traumatic brain injury is the main cause of mortality and disability in children and adult (Caveness, 1976; Kharlamove et al., 2011; Many and Barry, 2006). Although many studies have determined the relationship between TBI and PTE (Coulter et al., 1996; Golarai et al., 2001; Prince et al., 2009), the complex biological mechanism of epileptogenesis after brain injury remains controversial. Therefore, in this study, alteration of hippocampal interneurons (known to be critically involved to epileptogenesis) was investigated following TBI to understand abnormal neurotransmission at early stage. The results showed that immunohisto chemical distribution of parvalbumin (PV) and calbindin D-28k (CB) protein changed at specific time points after brain injury. These alterations revealed various expression patterns at different time.

To examine functional alteration of interneurons in the hippocampus following TBI, we used cryogenic TBI animal model and induced brain lesion to frontoparietal cortex by cooling cooper cone-shape cylinder (Fig.1). Immunoreactivities of PVpositive interneurons were observed in diverse interneuronal populations, including basket neurons, the axo-axonic neurons, and some interneurons near pyramidal cell layer and the dentate subgranule cell layer of the entire hippocampus (Figs. 2B1-B3). $\mathrm{CB}$ expression was mainly detected in the dentate granule cell layer and some interneurons of CA1 and CA2-3 (Fig. 3B1-B3). These expressional patterns of $\mathrm{PV}$ and $\mathrm{CB}$ immunoreactivity closely matched with the previous reports of immunohisto chemical distribution of CBPs (Celio, 1990). This isbecause PV is mainly contained in GABAergic interneurons such as basket cells and axo-axonic cells whereas CB is contained in granule cells located in the dentate gyrus and some neural cells (Toth and Freund, 1992; Hwang et al., 2004 a,b).

After 30 min of TBI, PV immunoreactivity was significantly reduced in CA1, CA2-3 and the hilar region of dentate gyrus than control (TBI 30 min, $4.5 \%$ in CA1, 5.8\% in CA2-3, 6.1\% in DG; control, $11.8 \%$ in CA1, 20.5\% in CA2-3, 18.7\% in DG; $P<0.01$, Figs. 2B1-B3, C1-C3, G and Table.1) (Kim et al., 2007b). Immunodensity analysis for PV expression also revealed significantly reduction at $30 \mathrm{~min}$ after TBI compared to that in the control groups (TBI $30 \mathrm{~min}, 75.2 \%$ in CA1, 78.4\% in CA2-3, $71.2 \%$ in DG; control, $100 \% ; P<0.01$, Fig. 2 F and Table.1) (Kim et al., 2007b). The expression of CB-positive interneurons also decreased, similar to PV immunoreactivity in the entire hippocampus (TBI 30 min, $15.8 \%$ in CA1, 7.8\% in CA2-3, 67.2\% in DG; control, $42.7 \%$ in CA1, $21.7 \%$ in CA2-3, 98.7\% in DG; $P<$ 0.01 and 0.05 , Fig. 3B1-B3, C1-C3, G and Table 2) (Kim et al., 2007b). In addition, its immunodensities were reduced (TBI 30 min, $65.3 \%$ in CA1, 56.7\% in CA2-3, 59.7\% in DG; control, $100 \%$; $P<0.01$, Fig. 3F) (Kim et al., 2007b). CBPs such as PV and CB are known as calcium buffers and facilitators of calcium diffusion in the nerve cell. They are widely utilized as excellent markers for specific inhibitory interneurons in the hippocampus (Celio, 1990; Andressen et al., 1993). In addition, enhanced intracellular calcium concentration due to various pathophysiological brain injuries can lead to diverse molecular changes related to neurodegeneration. Thus, declined expression of CBPs during this enhanced process of intracellular calcium levels can cause neuronal loss in brain disorders, including epilepsy (Magloczky et al., 1997; Fritschy et al., 1999; Sutch et al., 1999). Moreover, studies on epileptogenesis have reported that diverse interneuronal population containing CBPs are reduced in hippocampal complexes of epileptic patients (Magloczky et al., 2000; Gorter et al., 2001). Thus, reduced expression of CBPs is one specific marker for indicating neuronal degenerative phenotype in epileptic hippocampus (Fritschy et al., 1999; Sutch 

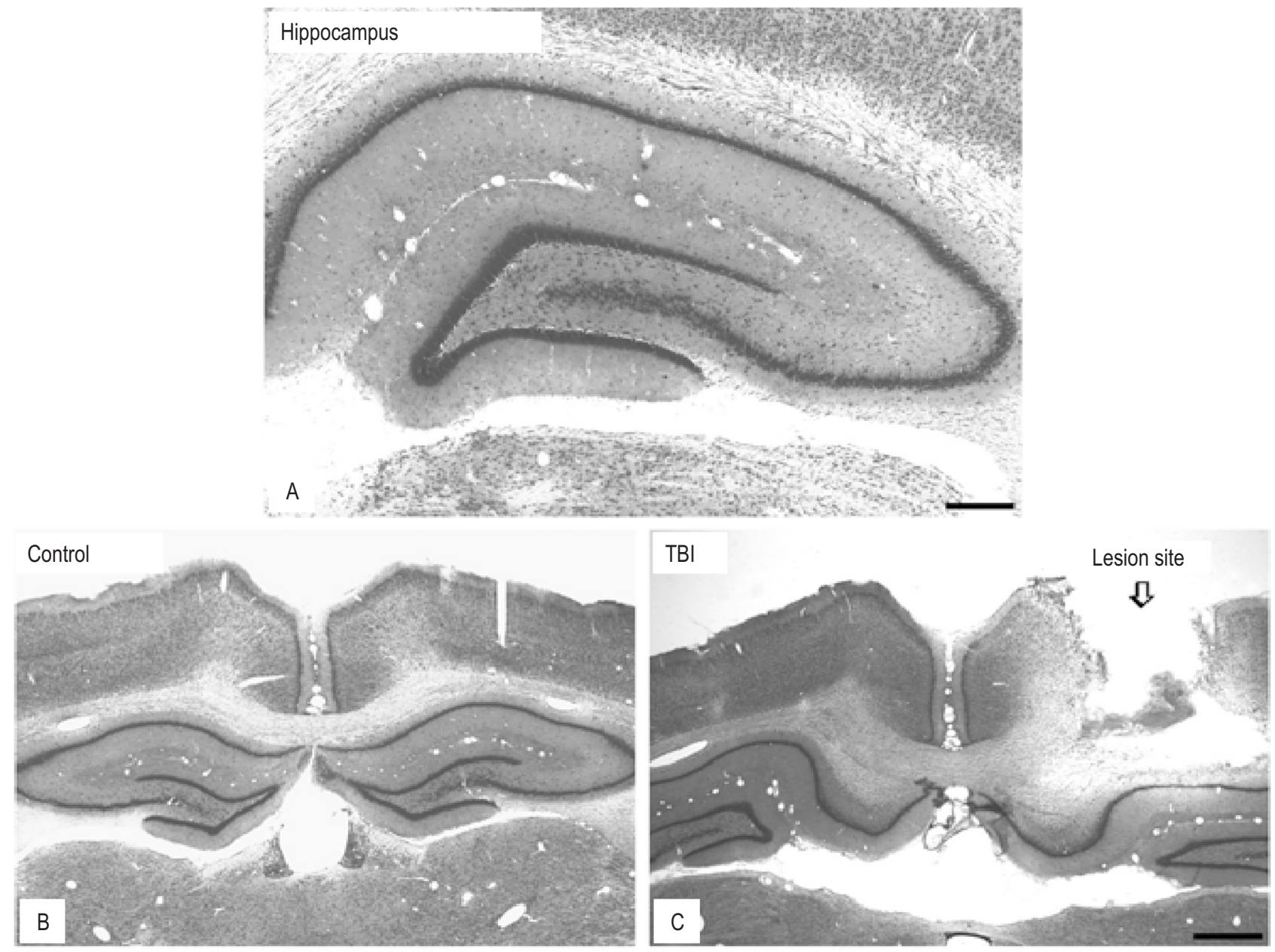

Fig. 1 : A representative photomicrograph of rat brain demonstrating loss of necrotic cavity at 1 day following a single episode of cryogenic brain injury in the left ipsilateral hemisphere. Neuronal loss of necrotic cavity was assessed with cresyl violet staining (A-C). A representative cresyl violet stained coronal section shows evidence of morphological changes in the ipsilateral cerebral cortex $(C)$ compared to the contralateral side (B). The open arrow in panel $\mathrm{C}$ represents the morphological alteration of cortical neurons at the cortical impact site. Bar $=280 \mu \mathrm{m}$ (panels $\mathrm{A}$ ) and $400 \mu \mathrm{m}$ (panels B and D).

Table 1 : Average cell numbers (mean \pm S.E.M per $250 \times 250>3^{2}$ ) of PV immunoreactive interneurons in lesioned hippocampus following traumatic brain injury

\begin{tabular}{llll}
\hline Traumatic brain injury & Region & Time course & Number of PV-positive interneurons \\
\hline Lesioned & CA1 & Control & $11.8 \pm 1.11$ \\
& 30 min & $4.5 \pm 0.72^{* *}$ \\
& 1 day & $10.6 \pm 1.39$ \\
& 3 day & $6.1 \pm 1.09^{* *}$ \\
& Control & $20.5 \pm 1.90$ \\
& CA2-3 & 1 day & $5.8 \pm 1.27^{* *}$ \\
& 3 day & $18.3 \pm 1.36$ \\
& Control & $17.9 \pm 1.83$ \\
& 30 min & 1 day & $18.7 \pm 2.37$ \\
& 3 day & $6.1 \pm 1.52^{* *}$ \\
& & $15.7 \pm 2.18$ \\
\end{tabular}

*Indicate significant differences from the control group, ${ }^{* *} P<0.01$ 

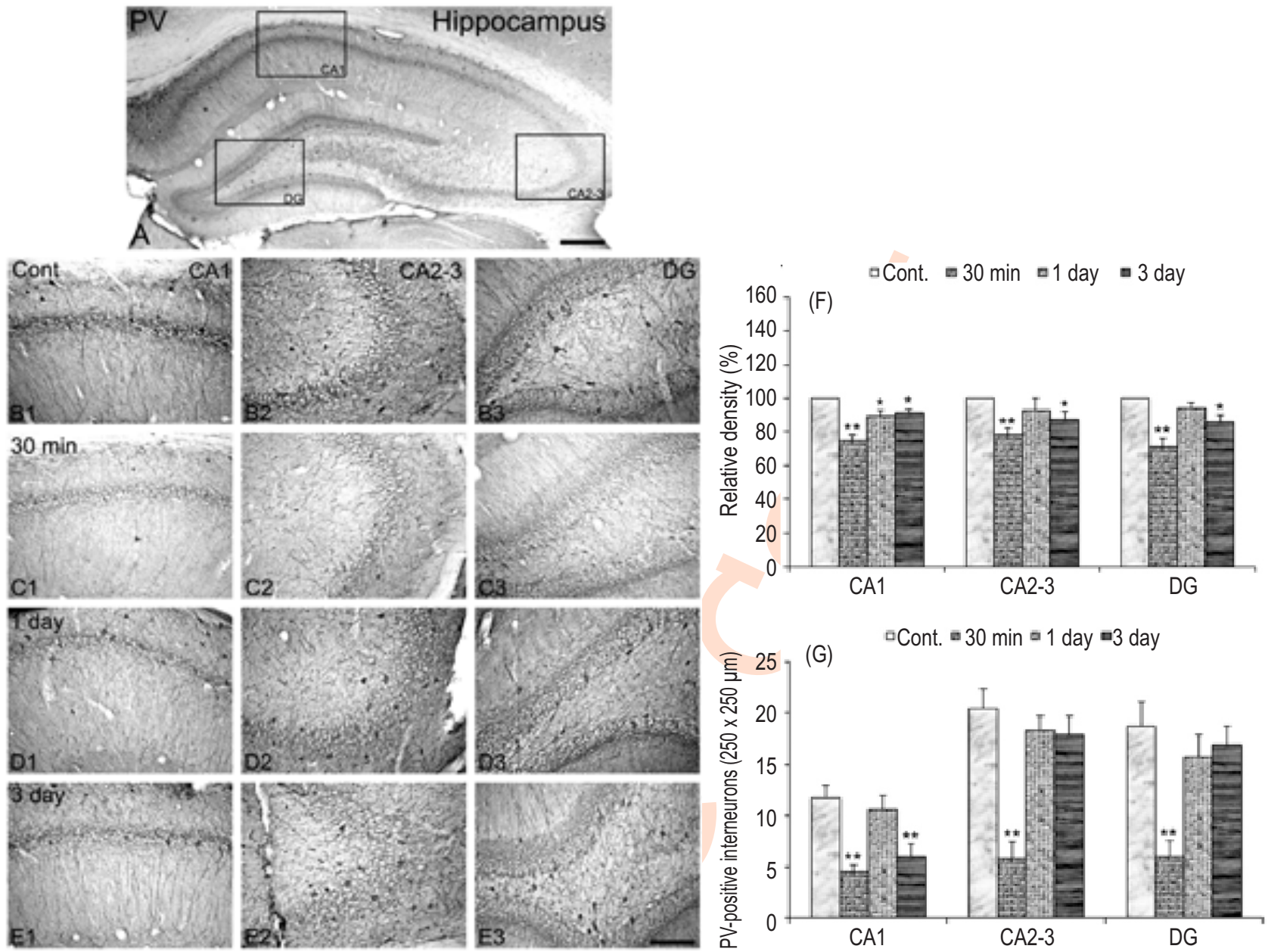

$(G)$

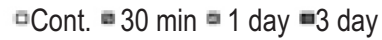

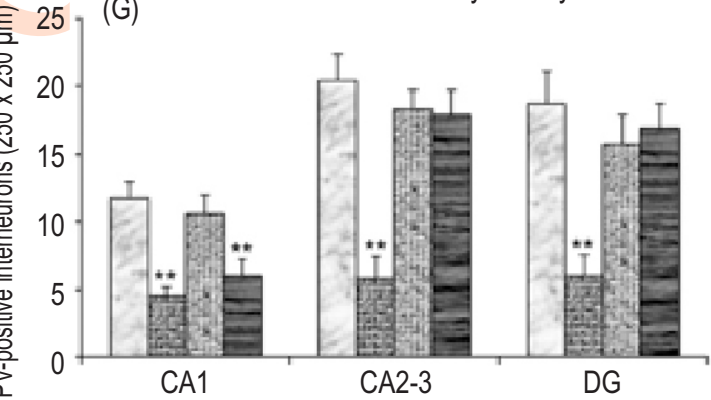

Fig. 2 : PV immunoreactivity in the ipsilateral hippocampus following cryogenic brain lesion. A representative photograph showing the normal hippocampal structure of brain (A). In the control animal, PV immunoreactive neurons are localized in the hilus and the subgranular cell layer of the dentate gyrus (B3). PV immunoreactive neurons are also abundantly observed in CA1 (B1) and CA2-3 region (B2). At 30 min after TBI, themarkedly reduced PV immunoreactive neurons are detected in all hippocampal regions (C1-C3). One day following TBI, although PV immunoreactivity is weakly enhanced, PV immunoreactive neurons are recovered to control levels (D1-D3). PV immunoreactive neurons at 3 day following TBI are unaltered in all region of lesioned hippocampus (E1-E3). Rectangles in panels A indicate the high-magnification of panels B1-B3, C1-C3, D1-D3 and E1-E3. Bars represent $280 \mu \mathrm{m}$ in $A$ and $50 \mu \mathrm{m}$ in B1-B3, C1-C3, D1-D3 and E1-E3. Quantitative analyses of PV immunoreactivities in lesioned hippocampus (F, mean \pm S.E.M). Significant differences from control hippocampus, ${ }^{*} P<0.05$ and ${ }^{* *} P<0.01$. Densitometric analyses show that $P$ Vexpression in lesioned hippocampus after TBI is changed depending on the time course compared to control hippocampus. Average cell numbers of PV immunoreactive interneurons in the lesioned hippocampus following TBI (G, mean \pm SD) are shown. Note that down-regulation of PV-positive interneurons in CA1 region at 3 day following TBI seems to be indicative of physical damage. Significant differences from the controls, ${ }^{* *} P<0.01$.

et al., 1999). Similar to previous investigations, we also observed significantly down-regulated PV and CB immunoreactivity in all hippocampus samples at 30 min following TBI. Such reduced immunohisto chemical expression of both proteins may reveal excessive increment of neuronal hyperexcitability in the hippocampus cause by declined inhibitory neurotransmission through GABAergic inhibitory interneurons. In fact, the diverse neuronal connections projected to hippocampal CA1 region are modulated by inhibitory influence of GABAergic interneurons (Empsonand Heinemann, 1995; Fritschy et al., 1999). Furthermore, epileptonesis in the hippocampus and cerebral cortex, following TBI is associated with excessive decline of postsynaptic inhibition and inhibitory interneurons (Zhu and Roper, 2000; Andre et al., 2001; Sayin et al., 2003). Excessive impairment of balance between excitatory and inhibitory neurotransmission caused by brain damage following TBI can lead to intense acceleration of neuronal hyperexcitability (Golarai et al., 2001; Graber et al., 2004; Prince et al., 2009). Thus, these processes may play an important role as epileptogenic factors after TBI. Therefore, results of this study suggested that deteriorated balance of neuronal excitation accompanied by inhibitory function of diverse interneurons at hippocampal CA1 

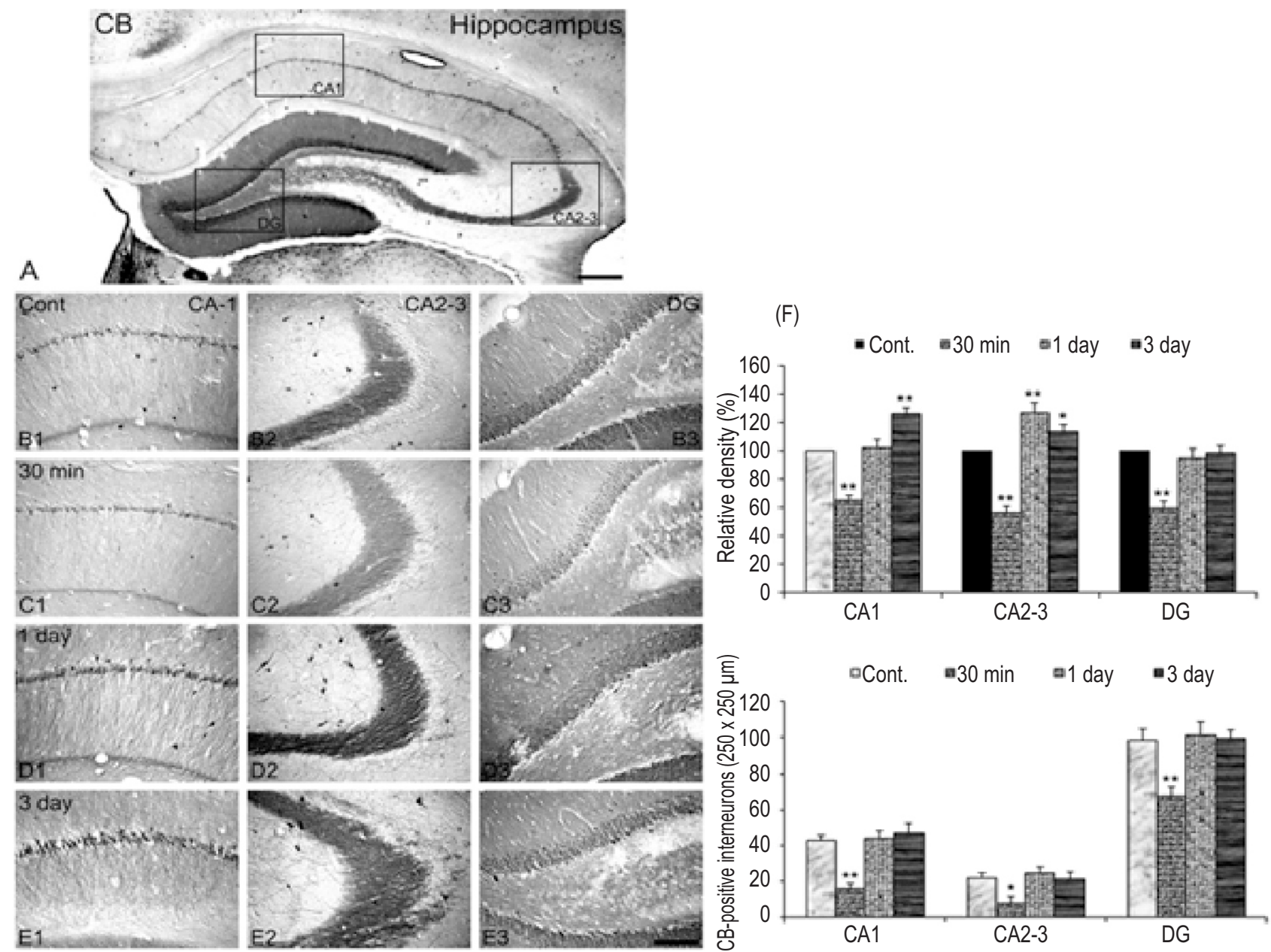

Fig. 3 : $\mathrm{CB}$ immunoreactivity in the ipsilateral hippocampus following TBI. A representative photograph shows the normal expression of CBimmunoreactivities in the hippocampus after TBI (A). In the control animal, CB immunoreactivity is mainly detected in the granular cell layer in the dentate gyrus (B3). In the CA1 region (B1), CB immunoreactivity is observed both in pyramidal cells and in interneurons. CB expression in the CA2-3 regions is detected in the mossy fibers as well as interneurons (B2). At 30 min after TBI, CB immunoreactivity is altered in all lesioned hippocampus compared to control levels (C1-C3). Although CB immunoreactivity in CA2-3 region at one day following TBI is enhanced over control levels, CB-positive interneurons are recovered to control levels (D1-D3). Three day following TBI, CB immunoreactiveinterneruons are unaltered in all region of lesioned hippocampus whereas its immunodensities in CA1 and CA2-3 are up-regulated compared to control levels (E1-E3). Rectangles in panels Aindicate the highmagnification of panels B1-B3, C1-C3, D1-D3 and E1-E3.Bar represent 400 $\mu \mathrm{m}$ in A and 50 $\mu \mathrm{m}$ in B1-B3, C1-C3, D1-D3 and E1-E3. Quantitative analyses of $C B$ immunoreactivities in lesioned hippocampus ( $F$, mean \pm S.E.M) are shown. Significant differences from control hippocampus, ${ }^{*} P<0.05$ and ${ }^{* *} P<$ 0.01.Densitometric analyses show that CBexpression in lesioned hippocampus following TBI are changed compared to control hippocampus, similar to immunohisthchemical data. Average cell numbers of $C B$ immunoreactive interneurons in lesioned hippocampus following TBI (G, mean \pm SD) are shown. Note that CB-positive neurons in the CA1-3 region at 30 min following TBI are down-regulated. Significant differences from controls, ${ }^{*} P<0.05$ and ${ }^{* *} P<0.01$.

region is caused by reduced $\mathrm{PV}$ and $\mathrm{CB}$ expression at this early time point after TBI, thus contributing to the development and spread of epileptogenic phenotype, following brain trauma.

On the other hand, the immunohistochemical distribution of PV-positive interneurons following TBI was recovered to control levels in all hippocampus regions (TBI 1 day, 10.6\% in CA1, 18.3\% in CA2-3, 15.7\% in DG; Fig. 2D1-D3, G and Table.1). PV immunoreactivity was maintained until 3 days after TBI, although its expression in CA1 decreased similar to that at $30 \mathrm{~min}$ after TBI (TBI 3 day, $6.1 \%$ in CA1, 17.9\% in CA2-3, 16.9\% in DG; Fig. 2E1-E3, G and Table.1). Quantitative data revealed some enhancement of $P V$ immunodensity at 1 day which maintained at 3 days, similar to control levels (TBI 1day, $89.7 \%$ in CA1, 92.7\% in CA2-3, 93.8\% in DG; TBI 3 days, $91.2 \%$ in CA1, 87.4\% in CA2$3,86.1 \%$ in DG; $P<0.05$, Fig. 2F) (Kim et al., 2007b). Moreover, the expression of CB-positive interneurons at 1 day was recovered in all hippocampal regions to levels similar to control (TBI 1 day, $44.1 \%$ in CA1, 24.8\% in CA2-3, 101.3\% in DG; Figs. 3D1-D3, G and Table.2). Its distribution was maintained until 3 
Table 2 : Average cell numbers (mean \pm S.E.M per $250 \times 250>3^{2}$ ) of CB immunoreactive interneurons in lesioned hippocampus following traumatic brain injury

\begin{tabular}{llll}
\hline Traumatic brain injury & Region & Time course & Number of CB-positive interneurons \\
\hline Lesioned & CA1 & Control & $42.7 \pm 3.21$ \\
& 30 min & $15.8 \pm 2.78^{* *}$ \\
& 1 day & $44.1 \pm 3.85$ \\
& 3 day & $47.2 \pm 4.92$ \\
& Control & $21.7 \pm 2.89$ \\
& 30 min & $7.8 \pm 3.79^{*}$ \\
& 1 day & $24.8 \pm 3.41$ \\
& 3 day & $21.1 \pm 4.24$ \\
& Control & 30 min & $98.7 \pm 6.07$ \\
& 1 day & $67.2 \pm 5.76^{* *}$ \\
& 3 day & $101.3 \pm 7.92$ \\
\hline
\end{tabular}

Footnote: Asterisks indicated significant differences from the control group, ${ }^{*} P<0.05,{ }^{* *} P<0.01$

days following TBI (TBI 3 days, $47.2 \%$ in CA1, 21.1\% in CA2-3, $99.7 \%$ in DG; Figs. 3E1-E3, G and Table.2). However, densitometric analysis of $\mathrm{CB}$ immunoreactivity showed enhanced expression in CA1 and CA2-3 at both time points, although its immunodensity was rehabilitated in the dentate gyrus, similar to control level after TBI (TBI 1day, 102.1\% in CA1, 126.7\% in CA2$3,94.6 \%$ in DG; TBI 3 days, $125.8 \%$ in CA1, $114.3 \%$ in CA2-3, $98.4 \%$ in DG; $P<0.05$ and 0.01, Fig. 3F) (Kim et al., 2007b). Previously, some investigators have demonstrated that the remaining interneurons in the epileptic hippocampus are exposed to hyperexcitation as compensational response to decreased GABAergic inhibition (Beau and Alger, 1998; Isokawa, 1998; Cossartet al., 2001). In addition, excessive abnormal response, including axonal sprouting in the injured specific brain region can appear at early time period until 3 days after TBI and such response can remain till one week (Yang et al., 1996; King et al., 2001). Thus, brain tissues injured by trauma should be treated within 3 days after TBI because delayed treatment can lead to deteriorating epileptogenesis in the brain (Graber and Prince, 2006). Moreover, interictal epileptiform activity in the damaged brain tissue is significantly enhanced at this early time points, hence revealing the occurrence of abnormal neural activity (Jacobs et al., 2001).Thus, the results of this study demonstrats that the re-enhanced immunohisto-chemical expression of PV and $\mathrm{CB}$ might be compensatory response to protect hippocampal neurons against diverse pathophysiological influences during epileptogenic processes after brain trauma. Therefore, inhibitory and excitatory neurotransmission ineffectiveness might occur at initial time after traumatic brain injury, leading to the development of epilepsy after TBI.

\section{Acknowledgments}

This work was supported by the Soonchunhyang University and a Basic Science Research Program (NRF2017R1D1A1B05036195) through National Research Foundation of Korea funded by the Ministry of Science.

\section{References}

Andre, V., C. Marescaux, A. Nehlig and J.M. Fritschy: Alterations of hippocampal GABAergic system contribute to development of spontaneous recurrent seizures in the rat lithium-pilocarpine model of temporal lobe epilepsy. Hippocam., 11, 452-468 (2001).

An, S.J. and D.S. Kim: Alterations in serotonin receptors and transporter immunoreactivities in the hippocampus in the rat unilateral hypoxic-induced epilepsy model. Cell Mol. Neurobiol., 31, 1245$1255(2011)$

Andressen, C., J. Blumcke and M.R. Celio: Calcium-binding proteins: Selective markers of nerve cells. Cell Tissue Res., 271, 181-208 (1993).

Beau, F.E and B.E. Alger: Transient suppression of GABAA-receptormediated IPSPs after epileptiform burst discharges in CA1 pyramidal cells. J. Neurophysiol., 79, 659-669 (1998).

Caveness, W.F.: Epilepsy, a product of trauma in our time. Epilepsia,17, 207-215(1976).

Celio, M.R.: Calbindin D-28k and parvalbumin in the rat nervous system. Neurosci., 35, 375-475 (1990).

Cossart, R., C. Dinocourt, J.C. Hirsch, A.M. Perez, J. De Felipe, Y. BenAri, M. Esclapez and C. Bernard: Dendritic but not somatic GABAergic inhibition is decreased in experimental epilepsy. Nat. Neurosci., 4, 52-62 (2001).

Coulter, D.A., A. Rafiq, M. Shumate, Q.Z. Gong, R.J. DeLorenzo and B.G. Lyeth: Brain injury-induced enhanced limbic epileptogenesis: Anatomical and physiological parallels to an animal model of temporal lobe epilepsy. Epilepsy Res., 26, 81-91 (1996).

De Lanerolle, N.C., J.H. Kim, R.J. Robbins and D.D. Spencer: Hippocampal interneuron loss and plasticity in human temporal lobe epilepsy. Brain Res., 495, 387-395 (1989).

Empson, R.M and U. Heinemann: The perforant path projection to hippocampal area CA1 in the rat hippocampal-entorhinal cortex combined slice. J. Physiol., 484, 707-720 (1995).

Fritschy, J.M., T. Kiener, V. Bouilleret and F. Loup: GABAergic neurons and GABAA-receptors in temporal lobe epilepsy. Neurochem. Int., $34,435-445$ (1999).

Gaetz, M.: The neurophysiology of brain injury. Clin. Neurophysiol., 115, 4-18(2004).

Garga, N. and D.H. Lowenstein: Posttraumatic epilepsy: Amajor problem 
in desperate need of major advances. Epilepsy Currents, 6, 1-5 (2006).

Golarai, G., A.C. Greenwood, D.M. Feeney and J.A. Connor: Physiological and structural evidence for hippocampal involvement in persistent seizure susceptibility after traumatic brain injury. J.Neurosci., 21, 8523-8537 (2001).

Gorter, J.A., E.A. van Vliet, E. Aronica and F.H. Lopes da Silva: Progression of spontaneous seizures after status epilepticus is associated with mossy fiber sprouting and extensive bilateral loss of hilar parvalbumin and somatostatin-immunoreactive neurons. Eur. J.Neurosci., 13, 657-669 (2001).

Graber, K.D and D.A. Prince: A critical period for prevention of posttraumatic neocortical hyperexcitability in rats. Ann. Neurol., 55, 860-870 (2004).

Graber, K.D. and D.A. Prince: Chronic partial cortical isolation. In: Models of seizures and epilepsy (Eds.: A. Pitkanen, P. Schwartzkroin and S. Moshe). Elsevier Academic Press, San Diego, pp.477-493 (2006).

Guerrini, R.: Idiopathic epilepsy and paroxysmal dyskinesia. Epilepsia, 423, 36-41 (2001).

Hwang, I.K., Y.S. Nam, D.W. Chung, H.S. Lee, Y.S. Yoon, K.Y. Yoo, T.C. Kang, I.S. Lee and M.H. Won: Changes in the expression of calbindin D-28k in the gerbil hippocampus following seizure. Neurochem. Int., 44, 145-152 (2004a).

Hwang, I.K., S.K. Park, S.J. An, K.Y. Woo, D.S. Kim, J.Y. Jung, M.H. Won, S.Y. Choi, O.S. Kwon and T.C. Kang: GABAA, not GABAB, receptor shows subunit- and spatial-specific alterations in the hippocampus of seizure prone gerbils. Brain Res.,1003, 98-107 (2004b).

Isokawa, M.: Modulation of GABAA receptor-mediated inhibiton by postsynaptic calcium in epileptic hippocampal neurons. Brain Res., 810, 241-250 (1998).

Jacobs, K.M., I. Parada and D.A. Prince: Enhanced c-fos staining in two post-lesional models of cortical hyperexcitability; freeze lesions and partial cortical isolations. Epilepsia,42, 221 (2001).

Kharlamove, E.A., E. Lepsveridze, M. Meparishvili, R.O. Solomonia, G. Lu, E.R. Miller, K.M. Kelly and Z.M. Mtchedlishvili: Alterations of GABAA and glutamate receptor subunits and heat shock protein in rat hippocampus following traumatic brain injury and in posttraumatic epilepsy. Epilepsy Res., 95, 20-34 (2011).

Kim, D.S., J.E. Kim, S.E. Kwak, M.H. Won and T.C. Kang: Seizure activity affects neuroglial Kv1 channel immunoreactivities in the gerbil hippocampus. Brain Res., 1151, 172-87 (2007a).

Kim, D.S., J.E. Kim, S.E. Kwak, H.C. Choi, H.K. Song, Y.I. Kim, S.Y. Choi and T.C. Kang: Up-regulated astroglial TWIK-related acidsensitive K+ channel-1 (TASK-1) in the hippocampus of seizuresensitive gerbils: A target of anti-epileptic drugs. Brain Res.,1185, 346-58 (2007b).

Kim, D.S., J.E. Kim, S.E. Kwak, K.C. Choi, D.W. Kim, O.S. Kwon, S.Y. Choi and T.C. Kang: Spatiotemporal characteristics of astroglial death in the rat hippocampo-entorhinal complex following pilocarpine-induced status epilepticus. J. Comp. Neurol., 511, 581-598 (2008).

King, C.E., A.J. Canty and J.C. Vickers: Alterations in neurofilaments associated with reactive brain changes and axonal sprouting following acute physical injury to the rat neocortex. Neuropathol. Appl.Neurobiol., 27, 115-126 (2001).
Magloczky, Z., P. Halasz, J. Vajda, S. Czirjak and T.F. Freund: Loss of calbinding-D28k immunoreactivity from dentate granule cells in human temporal lobe epilepsy. Neurosci., 76, 377-385 (1997).

Magloczky, Z., L. Wittner, Z. Borhegyi, P. Halasz, J. Vajda, S. Czirjak and T.F. Freund: Changes in the distribution and connectivity of interneurons in the pileptic human dentate gyrus. Neurosci., 96, 725 (2000).

Mani, J. and E. Barry: Posttraumatic epilepsy. In: The treatment of epilepsy: Principles and practice (Eds.: E. Wyllie, A. Gupta and D.K. Lachhwani). Hagerstown, MD: Lippincott Williams and Wilkins., pp.521-524 (2006).

Meldrum, B.: Do preclinical seizure models preselect certain adverse effects of antiepileptic drugs. Epilepsy Res., 50, 33-40 (2002).

Pagni, C.A. and F. Zenga: Posttraumatic epilepsy with special emphasis on prophylaxis and prevention. Acta. Neurochirurgica, 93, 27-34 (2005).

Peterson, G.M. and C.E. Ribak: Hippocampus of the seizure-sensitive gerbil is a specific site for anatomical changes in the GABAergic system. J. Comp. Neurol., 261, 405-422 (1987).

Prince, D.A., I. Parada, K. Scalise, K. Graber, X. Jin and F. Shen: Epilepsy following cortical injury: Cellular and molecular mechanisms as targets for potential prophylaxis. Epilepsia, 50, 30 40 (2009).

Rozycka, A. and W.H. Trzeciak: Genetic basis of autosomal dominant nocturnal frontal lobe epilepsy. J. Appl. Genet., 44, 197-207 (2003).

Sayin, U., S. Usting, J. Hagen, P. Rutecki and T. Sutula: Spontaneous seizures and loss of axo-axonic and axo-somatic inhibition induced by repeated brief seizures in kindled rats. J. Neurosci., 23, 2759-2768 (2003).

Siren, A.L., K. Radyushkin, S. Boretius, D. Kammer, C.C. Riechers, O. Natt, D. Sargin, T. Watanabe, S. Sperling, T. Michaelis, J. Price, B. Meyer, J. Frahm and H. Ehrenreich: Global brain atrophy after unilateral parietal lesion and its prevention by erythropoietin. Brain, 129, 480-489 (2006).

Sutch, R.J., C.C. Davies and N.G. Bowery: GABA release and uptake measured in crude synaptosomes from genetic absence epilepsy rats from Strasbourg (GAERS). Neurochem. Int., 34, 415-425 (1999).

Toth, K. and T.F. Freund: Calbindin D28k-containing nonpyramidal cells in the rat hippocampus; their immunoreactivity for GABA and projection to the medial septum. Neurosci., 49, 793-805 (1992).

Willmore, L.J. and Y. Ueda: Posttraumatic epilepsy: Hemorrhage, free radicals and the molecular regulation of glutamate. Neurochem. Res., 34, 688-697 (2009).

Weiss, G.H., D.M. Feeney, W.F. Caveness, D. Dillon, J.P. Kistler and J.P. Mohr: Prognostic factors for the occurrence of post-traumatic epilepsy. Arch. Neurol., 40, 7-10 (1983).

Weiss, G.H., A.M. Salazar, S.C. Vance, J.H. Grafman and B. Jabbari: Predicting posttraumatic epilepsy in penetrating head injury. Arch. Neurol., 43, 771-773 (1986).

Yang, Q., S. Wang, A. Hamberger and K.G. Haglid: Plasticity of granule cell-mossy fiber system following kainic acid induced seizures: An immunocytochemical study on neurofilament proteins. Neurosci. Res., 26, 57-64 (1996).

Zhu, W.J. and S.N. Roper: Reduced inhibition in an animal model of cortical dysplasia. J. Neurosci., 20, 8925-8931 (2000). 